\title{
Anomalias e variações na fórmula dentária em morcegos do gênero Artibeus Leach (Chiroptera, Phyllostomidae)
}

\author{
Ana Maria Rui ${ }^{1} \&$ César Jaeger Drehmer ${ }^{2}$ \\ ${ }^{1}$ Departamento de Zoologia, Universidade Federal do Rio Grande do Sul. Avenida Bento Gonçalves 9500, Bloco IV, \\ Prédio 43435, Sala 123, 91540-000 Porto Alegre, Rio Grande do Sul, Brasil. E-mail: ana.rui@ufrgs.br \\ 2 Departamento de Zoologia e Genética, Universidade Federal de Pelotas. Campus Universitário, Caixa Postal 354, \\ 96010-900 Pelotas, Rio Grande do Sul, Brasil. E-mail: cjaeger@terra.com.br
}

ABSTRACT. Anomalies and variation in the dental formula of bats of the genus Artibeus Leach (Chiroptera, Phyllostomidae). This paper describes and analyzes the causes of dental formula anomalies in the bats Artibeus lituratus (Olfers, 1818) and Artibeus fimbriatus Gray, 1838 (Phyllostomidae) belonging to populations inhabiting the southernmost Brazilian state of Rio Grande do Sul. Based on material examined and descriptions reported in the literature, is presented a general discussion on variation in the presence or absence of the third superior and inferior molars within members of the genus Artibeus (Leach, 1821). Of the 104 A. lituratus skulls examined was found one with an extra upper incisor and one with an extra upper right third molar, along with one case of lower third molar agenesis. In the $44 \mathrm{~A}$. fimbriatus skulls examined was found one with an extra upper right second premolar, a tooth which is usually absent in this species. The occurrence of the extra molar in A. lituratus and the second premolar in $A$. fimbriatus are atavistic, probably reflecting the ancestral $A$. lituratus in the case of the extra molar and, for the premolar, an even more ancient ancestor which lived before the divergence of the Phyllostomidae family. Regarding the genus as a whole, there is variation in the presence or absence of the third superior molar in practically all Artibeus species, such variation occurring at both the intrapopulational level and geographically. There is also already a low frequency of absent third inferior molars in various Artibeus species. Both superior and inferior third molars are in the process of exclusion from the dentition of Artibeus and do not occur in some species, and, when present, are always reduced in size and extremely simplified in their cuspidal pattern and do not participate in food processing: indicating a clear evolutionary trend to the loss of these teeth. Such variation is important because variability in the presence and absence of third molars means that these teeth are not appropriate characteristic for the identification of Artibeus species.

KEY WORDS. Agenesis, Artibeus fimbriatus, Artibeus lituratus, atavism, polymorphism.

RESUMO. Descreve-se a ocorrência e analisa-se as causas de anomalias dentárias em Artibeus lituratus (Olfers, 1818) e A. fimbriatus Gray, 1838 (Chiroptera: Phyllostomidae) provenientes de populações do Estado do Rio Grande do Sul, sul do Brasil. São discutidas, com base no material examinado e em ampla revisão da literatura, as variações quanto à presença dos terceiros molares superior e inferior entre diferentes espécies de Artibeus Leach, 1821. Foram analisados 104 crânios de $A$. lituratus e 44 de $A$. fimbriatus quanto à fórmula dentária. Em $A$. lituratus ocorreram dois casos de dentes extranumerários, um incisivo superior e um terceiro molar superior direito, e um de agênese dentária dos terceiros molares inferiores. Em $A$. fimbriatus constatou-se a ocorrência de um segundo pré-molar superior direito extranumerário. As ocorrências do terceiro molar superior em $A$. lituratus e do segundo pré-molar superior em $A$. fimbriatus são casos de atavismos. Em Artibeus (Artibeus) ocorrem variações quanto à presença do terceiro molar superior, de maior ou menor intensidade, em praticamente todas as espécies. Estas variações ocorrem tanto a nível intrapopulacional quanto geográfico. Já o terceiro molar inferior está ausente em baixa freqüência em várias populações de diferentes espécies. Os terceiros molares superiores e inferiores estão em processo de desaparecimento na linhagem dos Artibeus (Artibeus). O fato destes dentes já não ocorrerem em algumas espécies, terem ocorrência variável em outras e serem sempre estruturas reduzidas e simplificadas, sem função na mastigação, são indicativos deste processo evolutivo. A variação intensa observada quanto à ocorrência do terceiro molar superior inviabiliza o seu uso como caráter útil na identificação de espécies.

PALAVRAS CHAVE. Agênese, Artibeus fimbriatus, Artibeus lituratus, atavismo, polimorfismo. 
O gênero Artibeus Leach, 1821 tem distribuição restrita à Região Neotropical, ocorrendo desde o México, por toda a América Central e do Sul, até o norte da Argentina e sul do Brasil (Eisenberg \& Redford 1999, Koopman 1993). Algumas espécies de Artibeus estão entre os filostomídeos mais comuns e abundantes em comunidades Neotropicais. Por exemplo, no extremo sul do Brasil, A. lituratus (Olfers, 1818) e A. fimbriatus Gray, 1838 chegam a representar mais de 70\% dos morcegos coletados em áreas de Floresta Atlântica (Ruı \& Fabín 1997). Quanto aos hábitos alimentares, as espécies de Artibeus são primariamente frugívoras, mas podem consumir também pólen, néctar, partes de flores e insetos (GARDNER 1977).

Segundo Koopman (1993), o gênero Artibeus está dividido em quatro diferentes subgêneros: Artibeus, Dermanura Gervais, 1856, Enchistenes Andersen, 1906 e Koopmania Owen, 1991. Porém, não há consenso sobre o real status taxonômico destes grupos, que são tratados por outros autores como gêneros distintos (Bussche et al. 1998, Owen 1987, 1991, Wetterer et al. 2000). São aceitas, atualmente, por Koopman (1993) 17 espécies do gênero Artibeus: nove do subgênero Artibeus, seis do subgênero Dermanura, uma do subgênero Koopmania e uma do subgênero Enchistenes. A nível específico também ocorrem discrepâncias entre diferentes autores. Por exemplo, Handley (1987) considera $A$. planirostris (Spix, 1823) como uma subespécie de $A$. jamaicensis Leach, 1821, ao contrário de Koopman (1993) que a trata como uma espécie distinta.

A fórmula dentária das espécies do gênero Artibeus é I2/ 2, C1/1, P2/2, M2-3/2-3. As diferentes espécies apresentam número fixo de incisivos, caninos e pré-molares, porém, ocorre variação fenotípica quanto à presença do terceiro molar superior e inferior (EISENBERg \& REDFord 1999). A ausência do terceiro molar superior é considerada um caráter derivado para os filostomídeos (Wetterer et al. 2000).

Entre as espécies do subgênero Artibeus, ou seja, as espécies de "grandes" Artibeus, A. fimbriatus, A. fraterculus Anthony, 1924 e $A$. lituratus não apresentam o terceiro molar superior. Em A. jamaicensis, A. obscurus Schinz, 1821, A. inopinatus Davis \& Carter, 1964, A. hirsutus Anderse, 1906 e A. planirostris, o terceiro molar superior pode estar presente ou ausente. Artibeus amplus Handley, 1987 é a única espécie em que este molar está sempre presente. Todas as espécies deste subgênero possuem o terceiro molar inferior (Marques-Aguiar 1994). As espécies do subgênero Dermanura não apresentam os terceiros molares superiores e inferiores, ou então, possuem apenas o terceiro molar inferior, como em A. glaucus Thomas, 1893 (MuÑoz 1995). Em A. hartii Thomas, 1822 (subgênero Enchistenes), os terceiros molares superiores e inferiores são relativamente grandes, cerca de $35 \%$ do tamanho do segundo molar superior e inferior, respectivamente. Já em A. concolor Peters, 1865 (subgênero Koopmania) os terceiros molares superiores e inferiores são de tamanho médio, com cerca de $20 \%$ do tamanho do segundo molar superior e inferior, respectivamente (MARQUES-AgUIAR 1994).
A plasticidade fenotípica quanto à presença dos terceiros molares superiores em algumas espécies de Artibeus é conhecida, e a discussão sobre o tema gira em torno deste caráter ter ou não utilidade sistemática para a identificação das espécies. Porém, a questão de como esta variação se comporta entre diferentes espécies e entre diferentes populações de uma mesma espécie não foi explorada, ou seja, não há uma avaliação quantitativa sobre o assunto. Também não existem dados sobre os mecanismos causadores desta plasticidade fenotípica.

As fórmulas dentárias são diagnósticas e características para gêneros e espécies de mamíferos, sendo amplamente utilizadas na sistemática e taxonomia. Casos de alteração no número total de dentes têm sido relatados em vários gêneros de praticamente todas as ordens de mamíferos, incluindo tanto dentes ausentes como extranumerários (Wolsan 1984). Para o gênero Artibeus, há apenas um estudo disponível sobre anomalias dentárias em A. hirsutus, A. lituratus e A. phaeotis (Miller, 1902) do México (Ramírez-Pulido \& Müdespacher 1987). Não há estudos disponíveis para o Brasil ou outros países da América do Sul.

O presente estudo tem como objetivos: 1) descrever a ocorrência e analisar as causas de anomalias dentárias em Artibeus lituratus e A. fimbriatus provenientes de populações do Estado do Rio Grande do Sul, extremo sul do Brasil; 2) apresentar uma revisão e discutir, do ponto de vista evolutivo e filogenético, as variações quanto à presença ou não dos terceiros molares superiores e inferiores entre diferentes espécies e populações de Artibeus (Artibeus).

\section{MATERIAL E MÉTODOS}

Foram examinados 104 sincrânios de Artibeus lituratus, 48 machos e 56 fêmeas, e 44 de Artibeus fimbriatus, 19 machos e 25 fêmeas, provenientes de diferentes localidades do estado do Rio Grande do Sul, extremo sul do Brasil. Em cada um dos exemplares foram examinadas as arcadas dentárias do crânio e da mandíbula para análise da fórmula dentária.

O material examinado pertence às seguintes coleções científicas: Laboratório de Mamíferos, Departamento de Zoologia, Universidade Federal do Rio Grande do Sul, Porto Alegre (DZMAM); Museu de Ciências Naturais, Fundação Zoobotânica do Estado do Rio Grande do Sul, Porto Alegre (MCN) e; Coleção de Mastozoologia, Museu de Ciências Naturais, UNIVATES, Lajeado, Rio Grande do Sul (ZMUMCN).

Seguiu-se Koopman (1993) quanto à taxonomia e nomenclatura das espécies de Artibeus.

Adotou-se a convenção de letras maiúsculas (I, C, PM e M) seguidas por números sobrescritos, para indicar dentes superiores, e subscritos, para indicar dentes inferiores. A letra " $\mathrm{d}$ " precedente indica um dente decíduo. Quando necessário, também foram utilizadas fórmulas dentárias parciais, sendo que os dentes superiores são os indicados antes da barra e os inferiores depois da barra (Menu et al. 2002, Paula Couto 1979). 


\section{RESULTADOS}

Entre os 148 exemplares examinados foram constatadas quatro anomalias na fórmula dentária, três em $A$. lituratus e uma em $A$. fimbriatus. Os quatro exemplares portadores de anomalias dentárias foram coletados no município de Maquiné $\left(29^{\circ} 39^{\prime} 32^{\prime \prime} \mathrm{S}\right.$ e $\left.50^{\circ} 12^{\prime} 46^{\prime \prime} \mathrm{W}\right)$, que localiza-se no norte da Planície Costeira do Rio Grande do Sul, extremo sul do Brasil, em uma região de Floresta Atlântica (Floresta Ombrófila Densa). Cada uma das anomalias dentárias constatadas em A. lituratus tem freqüência de ocorrência de $0,96 \%(1 / 104)$ e a anomalia de $A$. fimbriatus tem freqüência de ocorrência de $2,27 \%(1 / 44)$ na região de estudo.

Em A. lituratus foram constatados dois casos de dentes extranumerários e um caso de agênese dentária. Em uma fêmea adulta (DZMAM 0062) foi constatada a presença do terceiro molar superior direito, que está ausente nesta espécie. Este dente é extremamente reduzido e simplificado, com coroa baixa e sem cúspides evidentes (Figs 1 e 2). Todos os demais exemplares de Artibeus examinados possuíam apenas dois molares superiores. O outro caso de dente extranumerário é a presença de um quinto incisivo superior em uma fêmea adulta (DZMAM 0100). O dente apresenta morfologia e tamanho semelhante aos incisivos característicos de Artibeus, porém é ligeiramente maior e mais transparente. Este dente, em vista oclusal, está em posição anterior à linha dos demais incisivos, exatamente entre o primeiro incisivo superior direito e o esquerdo (Figs 3 e 4). O terceiro caso de anomalia em A. lituratus foi a agênese do terceiro molar inferior em ambos os lados da mandíbula de um macho adulto (DZMAM 0060) (Fig. 5). Neste caso, os dentes e os alvéolos estão ausentes, descartando a hipótese de perda dentária de origem traumática, o que foi observado com certa freqüência no material examinado (Fig. 6). Todos os demais exemplares das duas espécies possuíam o terceiro molar inferior em ambos os lados da mandíbula.

Em $A$. fimbriatus constatou-se a ocorrência de um pequeno dente extranumerário, localizado entre o canino e o primeiro pré-molar superior direito em um macho adulto (DZMAM 0037). Este dente é reduzido em relação aos demais, possui coroa alta comprimida no sentido antero-posterior e apenas uma cúspide, não havendo sinais de cúspides acessórias (Figs 7 e 8).

$\mathrm{Na}$ tabela I são apresentados os dados obtidos na literatura sobre a variação quanto à presença dos terceiros molares superiores e inferiores em espécies do gênero Artibeus, subgênero Artibeus.

\section{DISCUSSÃO}

Anomalias dentárias podem ser de vários tipos: numéricas, morfológicas, de posição e oclusão, de erupção ou da estrutura do dente (Hoff \& Hoff 1996). No presente estudo, todas as quatro anomalias constatadas são do tipo numéricas, três envolvendo dentes extranumerários e uma envolvendo uma agênese dentária. Apesar das causas das anomalias dentárias muitas vezes serem de difícil comprovação, pode-se tecer algumas hipóteses a este respeito.

\section{Incisivo extranumerário}

O caso do incisivo extranumerário (DZMAM 0100), provavelmente, está relacionado a um distúrbio ontogenético de duplicação do germe dentário de um incisivo (o I ${ }^{1}$ esquerdo ou o direito), levando à formação de um dente extra. A semelhança morfológica e de tamanho entre este dente e os demais reforça esta hipótese. A outra possibilidade é a deste dente ser um decíduo $\left(\mathrm{dI}^{1}\right)$. Entretanto, sua morfologia não é típica dos dentes decíduos de quirópteros em geral, que possuem um formato estiliforme, adaptado ao ato de agarrar-se ao pêlo e ao mamilo da mãe quando ainda em fase de lactação (Sigé et al. 1998).

Casos de incisivos extranumerários são conhecidos em vários grupos de mamíferos (Wolsan 1984, Hoff \& Hoff 1996, Zima 1988). Em Artibeus, há um caso de incisivo extranumerário inferior descrito para A. hirsutus no México (Farney in Webster \& JoNEs 1983). Especificamente para $A$. lituratus, há três casos registrados para exemplares do México: uma fêmea adulta com um incisivo a mais entre o $\mathrm{I}_{2}$ e o canino em cada lado da mandíbula; um macho com um incisivo a mais entre o $\mathrm{I}^{2}$ e o canino na maxila direita; e um macho adulto com dois incisivos extranumerários na maxila em contato com os I ${ }^{1}$ (RAMíREz-PuLIDO \& Müdespacher 1987).

\section{Atavismo do terceiro molar superior}

A presença do terceiro molar superior direito na fêmea adulta de $A$. lituratus (DZMAM 0062) é um caso de atavismo espontâneo. HaLl (2003) define atavismos (do latim "atavus" que significa avô do avô do avô) como "uma característica que esteve presente em linhagens ancestrais mais distantes e que aparece em baixa freqüência em membros individuais de uma população, usualmente em um ou poucos indivíduos".

As características dessa ocorrência enquadram-se nos quatro critérios básicos para o reconhecimento de um atavismo (segundo Hall 1984): 1. persistência da característica na vida adulta (o exemplar é uma fêmea adulta); 2 . ausência desta característica nos pais ou em ancestrais recentes (A. lituratus normalmente não apresenta este dente); 3 . presença em somente um ou poucos indivíduos dentro de uma população $(0,96 \% ; 1 /$ 104) e 4. semelhança ou identidade com o mesmo caráter apresentado por todos os membros da população ancestral. $\mathrm{O} \mathrm{M}^{3}$ atávico da fêmea de $A$. lituratus tem posição e morfologia praticamente iguais aos terceiros molares superiores das espécies de Artibeus que normalmente, ou geralmente, possuem este dente. Observe a semelhança do $\mathrm{M}^{3}$ atávico de A. lituratus (Figs 1 e 2) com os $\mathrm{M}^{3}$ de $A$. obscurus (Figs 9 e 10), espécie que normalmente apresenta este dente.

Quando é considerada a filogenia das espécies do subgênero Artibeus, se observa que $A$. lituratus é a espécie mais derivada, e que seu grupo irmão inclui espécies que podem possuir ou não os terceiros molares superiores (MARQues-Aguiar 1994). Desta forma, há vários indícios de que o $\mathrm{M}^{3}$ desta fêmea seja o 

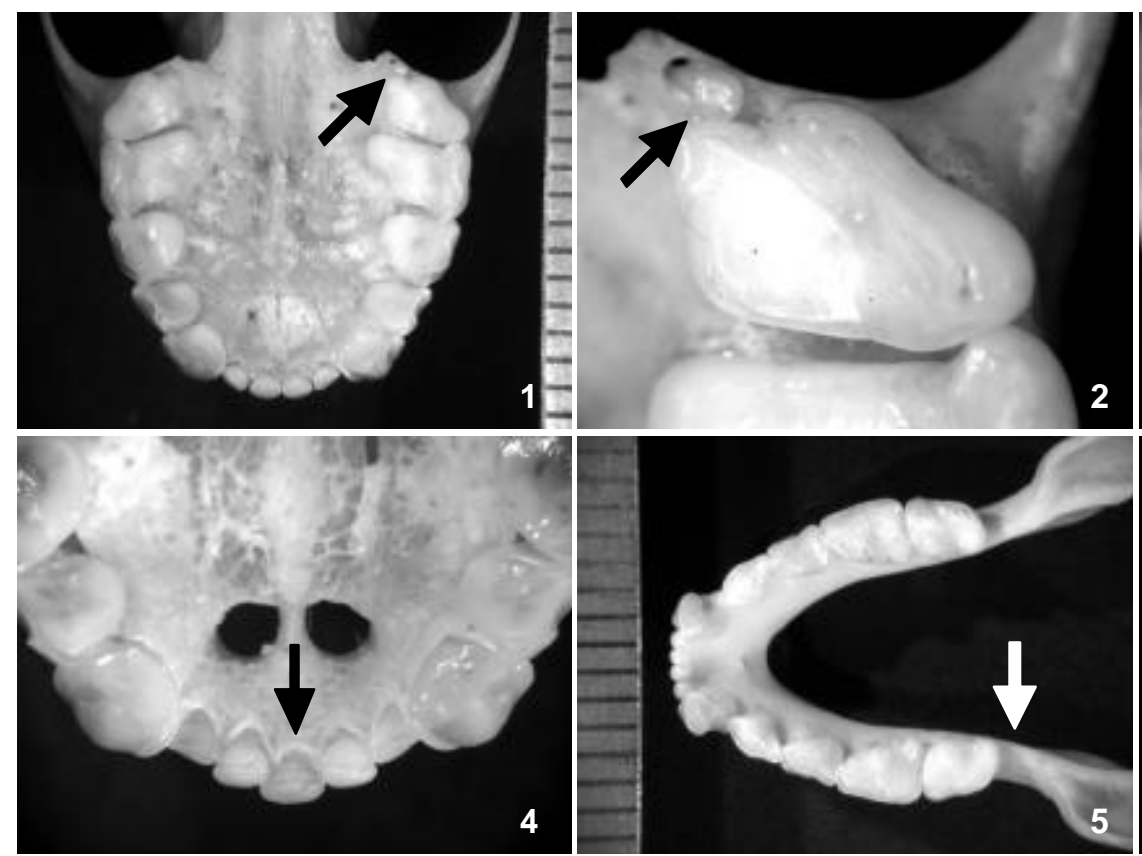
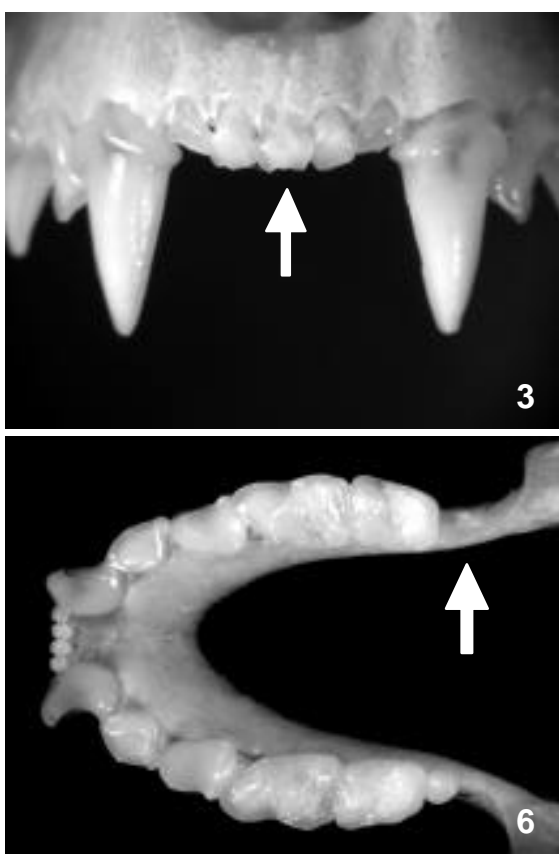

Figuras 1-6. (1-2) Artibeus lituratus (DZM AM 0062): (1) vista palatal da série dentária, (2) detalhe do terceiro molar superior atávico; (34) Artibeus lituratus (DZM AM 0100): (3) vista anterior, (4) palatal do incisivo extranumerário; (5) Artibeus lituratus (DZMAM 0060), vista oclusal da mandíbula com a agênese dos terceiros molares inferiores; (6) Artibeus lituratus (DZMAM 0100), vista oclusal da mandíbula com o terceiro molar inferior esquerdo e o alvéolo no lado direito. Escala das fotos em milímetros.
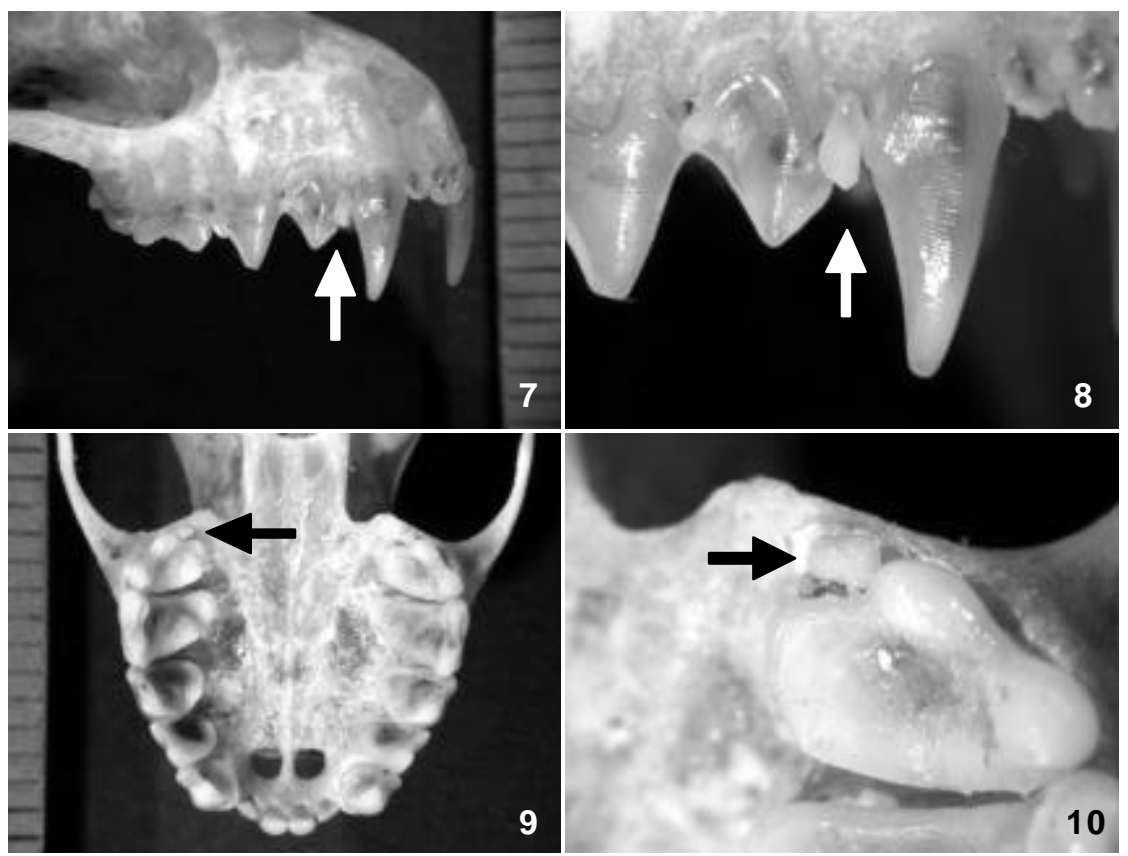

Figuras 7-10. (7-8) Artibeus fimbriatus (DZMAM 0037): (7) vista lateral da região anterior do crânio, (8) detalhe do pré-molar extranumerário; (9-10) Artibeus obscurus (DZM AM 0679): (9) vista palatal da série dentária, (10) detalhe do terceiro molar superior. Escala das fotosem milímetros.

Revista Brasileira de Zoologia 21(3): 639-648, setembro 2004 
Tabela I. Revisão das informações sobre a ocorrência dos terceiros molares superiores e inferiores em espécies de Artibeus (Artibeus) na região Neotropical. (N) Número total de exemplares examinados, $(n)$ número de exemplares examinados com determinada fórmula dentária, $\left(M^{3}\right)$ terceiro molar superior, $\left(M_{3}\right)$ terceiro molar inferior, $(M 2 / 2, M 2 / 3, M 3 / 2$ e M3/3) número de molares superiores e inferiores.

\begin{tabular}{|c|c|c|}
\hline Espécie/ fonte & Localidade & Informação \\
\hline \multicolumn{3}{|l|}{ A. fimbriatus Gray, 1838} \\
\hline MyeRs \& WetZel (1979: 639) & Paraguai & $\mathrm{N}=11 ; \mathrm{M}^{3}$ ausente \\
\hline BARQUEZ et al. (1999: 66-67) & Argentina e Paraguai & $\mathrm{N}=8 ; \mathrm{M}^{3}$ ausente \\
\hline DIAs et al. (2002: 126) & Rio de Janeiro, Brasil & $\mathrm{N}=32 ; \mathrm{M}^{3}$ ausente \\
\hline SÉrGıo Althoff (com. pes.) & Santa Catarina e Paraná, Brasil & $\mathrm{N}=57 ; \mathrm{n}=56(98,25 \%) \mathrm{M}^{3}$ ausente \\
\hline Presente trabalho & Rio Grande do Sul, Brasil & $N=44 ; M 2 / 3$ \\
\hline \multicolumn{3}{|l|}{ A. fraterculus Anthony, 1924} \\
\hline D.R. Patten (1971, dados não publicados) & Equador e Peru & $N=38 ; n=37(97,4 \%) M 2 / 3 ; n=1(2,6 \%) M 2 / 2$ \\
\hline \multicolumn{3}{|l|}{ A. hirsutus K. Andersen, 1906} \\
\hline ANDERSON in WeBster \& JONES JR. (1983: 1) & México & $\begin{array}{l}\mathrm{N}=88 ; \mathrm{n}=76(86,4 \%) \mathrm{M} 3 / 3 ; \mathrm{n}=1(1 \%) \mathrm{M} 2 / 2 \\
\mathrm{n}=8(9,1 \%) \mathrm{M} 2 / 3 ; \mathrm{n}=3(3,4 \%) \mathrm{M}^{3} \text { ausente de } \\
\text { um lado }\end{array}$ \\
\hline \multicolumn{3}{|l|}{ A. inopinatus Davis \& Carter, 1964} \\
\hline Burt \& StiRTON in Webster \& JOnes JR. (1983: 1) & El Salvador & $\mathrm{N}=5 ; \mathrm{n}=4(80 \%) \mathrm{M} 3 / 3 ; \mathrm{n}=1(20 \%) \mathrm{M}^{3}$ ausente \\
\hline \multicolumn{3}{|l|}{ A. jamaicensis Leach, 1821} \\
\hline \multirow[t]{2}{*}{ Davis (1970: 110) } & México (Pacífico) & $N=71 ; n=70(98,6 \%) M 3 / 3$ \\
\hline & América Central & $\begin{array}{l}\mathrm{N}=725 ; \mathrm{n}=691(95,3 \%) \mathrm{M} 2 / 3 ; \mathrm{n}=10(1,4 \%) \\
\mathrm{M} 2 / 2 ; \mathrm{n}=7(1 \%) \mathrm{M} 2 / 2 \mathrm{em} \text { um lado e } \mathrm{M} 2 / 3 \mathrm{no} \\
\text { outro; } \mathrm{n}=9(1,2 \%) \mathrm{M} 2 / 3 \text { em um lado e } \mathrm{M} 3 / 3 \text { do } \\
\text { outro; } \mathrm{n}=8(1,1 \%) \mathrm{M} 3 / 3\end{array}$ \\
\hline D.R. Patten (1971, dados não publicados) & Equador (A. j. aequatorialis) & $\begin{array}{l}\mathrm{N}=11 ; \mathrm{n}=9(82 \%) \mathrm{M} 2 / 3 ; \mathrm{n}=1 \text { (9\%) M3/3 só de } \\
\text { um lado; } \mathrm{n}=1(9 \%) \mathrm{M} 3 / 2\end{array}$ \\
\hline \multirow[t]{3}{*}{ JONES JR. (1978: 3-4) } & $\begin{array}{l}\text { Barbados e St. Lucia (A. j. } \\
\text { jamaicensis) }\end{array}$ & $\mathrm{N}=24 ; \mathrm{M}^{3}$ ausente \\
\hline & St. Vincent $(A . j$. schwartzi) & $\begin{array}{l}\mathrm{N}=39 ; \mathrm{n}=4(10 \%) \mathrm{M}^{3} \text { presente; } \mathrm{n}=1(2,5 \%) \mathrm{M}_{3} \\
\text { ausente de um lado }\end{array}$ \\
\hline & $\begin{array}{l}\text { Grenada e Trinidad ( } A . j . \\
\text { trinitatis) }\end{array}$ & $\begin{array}{l}\text { Grenada: } n=18(94 \%) M^{3} \text { presente Trinidad: } n= \\
40(100 \%) M^{3} \text { presente }\end{array}$ \\
\hline MyeRs \& Wetzel (1979: 639) & Paraguai & $\mathrm{N}=33 ; \mathrm{M}^{3}$ presente \\
\hline TABOADA (1979: 188) & Cuba (A. j. parvipes) & $\mathrm{N}=146 ; \mathrm{n}=136(93 \%) \mathrm{M} 2 / 3 ; \mathrm{n}=10(7 \%) \mathrm{M} 2 / 2$ \\
\hline TADDEI (1979: 911) & São Paulo, Brasil (A. j. planirostris) & $\mathrm{N}=147 ; \mathrm{M}^{3}$ presente \\
\hline ANDERSON et al. (1982: 8) & Bolívia & Presença $\mathrm{x}$ ausência do $\mathrm{M}^{3}$ variável individualmente \\
\hline \multirow[t]{2}{*}{ HANDLEY (1987: 164) } & Leste dos Andes & $\mathrm{M} 3 / 3$ \\
\hline & $\begin{array}{l}\text { Oeste dos Andes e América } \\
\text { Central }\end{array}$ & $\mathrm{M} 2 / 3$ \\
\hline SIMMONS \& Voss (1998: 101) & Paracou, Guiana Francesa & $\mathrm{N}=35 ; \mathrm{M}^{3}$ presente \\
\hline \multirow[t]{2}{*}{ TADDEl et al. (1998: 100 e 106) } & São Paulo, Brasil & $N=40 ; M 3 / 3$ \\
\hline & Piauí, Brasil & $N=30 ; M 3 / 3$ \\
\hline SÉRGIO Althoff (com. pes.) & Santa Catarina e Paraná, Brasil & $\mathrm{N}=9 ; \mathrm{n}=8(90 \%) \mathrm{M}^{3}$ ausente \\
\hline \multicolumn{3}{|l|}{ A. lituratus (Olfers, 1818) } \\
\hline DAVIS (1970: 107) & América Central & $N=201 ; M 2 / 3$, com uma exceção \\
\hline D.R. Patten (1971, dados não publicados) & $\begin{array}{l}\text { América Central e do Sul (vários } \\
\text { países) }\end{array}$ & $\begin{array}{l}N=338 ; n=337(99,8 \%) M 2 / 3 ; n=1(0,2 \%) \\
M 2 / 2\end{array}$ \\
\hline MyeRs \& Wetzel (1979: 639) & Paraguai & $\mathrm{N}=34 ; \mathrm{M}^{3}$ ausente \\
\hline TAdDel (1979: 911) & São Paulo, Brasil & $\mathrm{N}=169 ; \mathrm{M}^{3}$ ausente \\
\hline WEBSTER \& JONES JR. (1980: 5) & Bolívia & $\mathrm{N}=12 ; \mathrm{M}^{3}$ ausente \\
\hline
\end{tabular}


Tabela I. Continuação.

\begin{tabular}{|c|c|c|}
\hline Espécie/ fonte & Localidade & Informação \\
\hline \multicolumn{3}{|l|}{ A. lituratus (Olfers, 1818) (continuação) } \\
\hline ANDRson et al. (1982: 9) & Bolívia & $\mathrm{M}^{3}$ ausente \\
\hline DAVIS (1984: 10) ${ }^{2}$ & $\begin{array}{l}\text { México e América Central (vários } \\
\text { países) }\end{array}$ & $\begin{array}{l}\mathrm{M} 2 / 3 ; \mathrm{M}_{3} \text { ausente em um ou ambos os lados em } \\
\text { cerca de } 2 \% \text { da população }\end{array}$ \\
\hline DAVIS (1984: 13) & $\begin{array}{l}\text { México, América Central, } \\
\text { Colômbia e Venezuela (A. I. } \\
\text { palmarum) }\end{array}$ & $\begin{array}{l}N=150 ; n=149(99,3 \%) M 2 / 3 ; n=1(0,7 \%) \\
M 2 / 2\end{array}$ \\
\hline KOEPCKe \& KRAFT (1984: 79) & Peru & $\mathrm{N}=13 ; \mathrm{M}^{3}$ ausente \\
\hline RAMíREZ-Pulido \& MüDESACHER (1987: 35-41)1 & México & $\begin{array}{l}\mathrm{N}=409 ; \mathrm{n}=1(0,24 \%) \mathrm{M}^{3} \text { presente de um lado; } \mathrm{n} \\
=2(0,49 \%) \mathrm{M}_{3} \text { ausente em ambos os lados }\end{array}$ \\
\hline Simmons \& Voss (1998: 101) & Paracou, Guiana Francesa & $\mathrm{N}=24 ; \mathrm{M}^{3}$ ausente \\
\hline BARQUEZ et al. (1999: 68) & Argentina & $\mathrm{N}=45 ; \mathrm{M} 2 / 3$ \\
\hline Ś́rgio Althor (com. pes.) & Santa Catarina e Paraná, Brasil & $\mathrm{N}=182 ; \mathrm{n}=177(97,25 \%) \mathrm{M}^{3}$ ausente \\
\hline Presente trabalho & Rio Grande do Sul, Brasil & $\begin{array}{l}\mathrm{N}=104 ; \mathrm{n}=1(0,67 \%) \mathrm{M}^{3} \text { presente de um lado; } \mathrm{n} \\
=1(0,67 \%) \mathrm{M}_{3} \text { ausente ambos os lados }\end{array}$ \\
\hline \multicolumn{3}{|l|}{ A. obscurus Schinz, 1821} \\
\hline D.R. Patten (1971, dados não publicados)² & Colômbia, Equador e Peru & $N=42 ; n=38(91 \%) M 3 / 3 ; n=4(9 \%) M 2 / 3$ \\
\hline WMSTRR \& JONES JR. $(1980: 5)^{3}$ & Bolívia & $\mathrm{N}=19 ; \mathrm{M}^{3}$ presente \\
\hline KOEPCKE \& KRAFT (1984: 79)³ & Peru & $\mathrm{N}=13 ; n=12(92,3 \%) \mathrm{M} 3 / 3 ; \mathrm{n}=1(7,7 \%) \mathrm{M} 2 / 3$ \\
\hline \multirow[t]{3}{*}{ HANDLEY (1989: 451) } & Amapá, Brasil; Venezuela & $\begin{array}{l}\mathrm{N}=193 ; \mathrm{M}^{3} \text { presente }(94 \%) ; 6 \% \text { ausente em um } \\
\text { ou ambos os lados }\end{array}$ \\
\hline & Colômbia, Equador e Peru & $\begin{array}{l}\mathrm{N}=31 ; \mathrm{M}^{3} \text { presente }(94 \%) ; 6 \% \text { ausente em um ou } \\
\text { ambos os lados }\end{array}$ \\
\hline & Sul da Amazônia, Brasil & $\begin{array}{l}\mathrm{N}=26 ; \mathrm{M}^{3} \text { presente }(77 \%) ; 19 \% \text { ausente em um } \\
\text { ou ambos os lados }\end{array}$ \\
\hline Simmons \& Voss (1998: 103) & Paracou, Guiana Francesa & $\mathrm{N}=34 ; \mathrm{n}=3(8,8 \%) \mathrm{M}^{3}$ ausente \\
\hline \multirow[t]{2}{*}{ TADDEl et al. (1998: 100) } & São Paulo até Paraíba, Brasil & $\mathrm{N}=24 ; \mathrm{M} 2 / 3$ \\
\hline & Oeste da Amazônia, Brasil & $\mathrm{N}=31 ; \mathrm{n}=30(96,77 \%) \mathrm{M}^{3}$ presente \\
\hline DiAs et al. (2002: 128) & Rio de Janeiro, Brasil & $\begin{array}{l}\mathrm{N}=10 ; \mathrm{n}=8(80 \%) \mathrm{M}^{3} \text { ausente; } \mathrm{n}=1(10 \%) \mathrm{M}^{3} \\
\text { presente; } \mathrm{n}=1(10 \%) \mathrm{M}^{3} \text { presente de um lado }\end{array}$ \\
\hline Ś́rgio Althof (com. pes.) & Santa Catarina e Paraná, Brasil & $\mathrm{N}=11 ; \mathrm{n}=10(90,91 \%) \mathrm{M}^{3}$ ausente \\
\hline \multicolumn{3}{|l|}{ A. planirostris (Spinx, 1823) } \\
\hline D.R. Patten (1971, dados não publicados) & $\begin{array}{l}\text { Guiana, Suriname, Guiana } \\
\text { Francesa, Peru e Brasil }\end{array}$ & $N=48 ; n=44(92 \%) M 3 / 3 ; n=4(8 \%) M 2 / 3$ \\
\hline WBSTRR \& JONES JR. (1980: 5) & Bolívia & $\mathrm{N}=15 ; \mathrm{M}^{3}$ presente \\
\hline ANDRSON et al. (1982: 9) & Bolívia & $\mathrm{M}^{3}$ presente \\
\hline KOEPCKE \& KRAFT (1984: 79) & Peru & $N=36 ; n=35(97 \%) M 3 / 3 ; n=1(3 \%) M 2 / 3$ \\
\hline BARQUEZ et al. (1999: 73) & Argentina (A. p. fallax) & M2-3/3; "M³ pequeno e geralmente presente" \\
\hline
\end{tabular}

1) Artibeus lituratus é tratado por estes autores como A. intermedius J.A. Allen, que foi considerado por KoopmAN (1993) como sinonímia de A. lituratus; 2) Artibeus obscurus foi descrito por este autor em sua tese como A. davisi, porém este trabalho nunca foi publicado e A. davisi é considerado por H ANDLEY (1989) como nomen nudum; 3) Artibeus obscurus étratado por estes autores como A. fuliginosus. HANDLEY (1989) considerou esta denominação inválida e redescreveu a espécie, sendo que atualmente a denominação A. obscurus é amplamente aceita e citada.

mesmo dente presente nas outras espécies do subgênero, que foi perdido em A.lituratus, e que reaparece em baixíssima freqüência em al guns indivíduos de certas populações, o que caracteriza um atavismo.

A presença do terceiro molar superior em A. lituratus é extremamente rara e está registrada apenas para exemplares coletadosno sul do Brasil e para uma fêmea adulta coletada no México (Tab. I). No material examinado, proveniente do Rio Grande do Sul, apenas um indivíduo $(0,67 \%)$ possuía o terceiro molar superior. Já em Santa Catarina, de 182 exemplares exami- 
nados, cinco $(2,75 \%)$ possuíam este dente (comunicação pessoal Sérgio Althoff). Além disso, há um caso de presença de terceiro molar superior para A. fimbriatus em Santa Catarina (comunicação pessoal Sérgio Althoff) (Tab. I). A ocorrência destes atavismos deve ser melhor investigada e pode estar ligada a características genéticas particulares destas populações de Artibeus.

Casos de atavismos espontâneos são raros na literatura, mas incluem exemplos clássicos como a ocorrência de dedos extranumerários nos cavalos (Gould 1982), o reaparecimento dos membros locomotores posteriores em alguns grupos de cetáceos (BEjDER \& HALL 2002) e ainda o ressurgimento dos terceiros molares inferiores nos linces, Lynx lynx (WEDERLIN 1987). Casos de atavismos espontâneos ocorrem ainda em outros grupos de vertebrados e invertebrados (Hall 1984, STIASSNy 1992, 2002).

\section{Atavismo do segundo pré-molar}

No caso do dente extranumerário do exemplar DZMAM 0037, várias possibilidades devem ser consideradas. Este dente, a exemplo daquele do espécime DZMAM 0100, poderia ser considerado um decíduo $\left(\mathrm{dPM}^{3}\right)$, retido até a vida adulta, mas sua morfologia não se assemelha a um pré-molar decíduo, conforme já discutido anteriormente. A análise de um feto de Artibeus (MCN 2415), com a dentição decídua ainda presente, corrobora essa informação, descartando a hipótese de que este dente extranumerário seja decíduo.

Nenhum quiróptero, recente ou fóssil, possui mais do que três pré-molares superiores, e estes são sempre o $\mathrm{PM}^{2}, \mathrm{PM}^{3}$ e $\mathrm{PM}^{4}$. Os dois pré-molares superiores de Artibeus são, evolutivamente, o $\mathrm{PM}^{3}$ e PM${ }^{4}$ (Wetterer et al. 2000), logo se pode supor que este dente seja produto de uma alteração ontogenética levando à duplicação do germe dentário do $\mathrm{PM}^{3}$, devido à posição que este dente ocupa na série dentária (Figs 7 e 8). A hipótese de alterações no desenvolvimento gerando duplicações dentárias foi defendida por alguns autores (Wolsan 1984, Astúa de Moraes et al. 2001), que atribuíram a duplicação a fatores como mutação ou distúrbios no controle gênico do desenvolvimento dentário.

Entretanto, a hipótese mais plausível refere-se à possibilidade de um outro caso de atavismo espontâneo, nesse caso um $\mathrm{PM}^{2}$ atávico, considerando-se a presença normal do $\mathrm{PM}^{3} \mathrm{e}$ do $\mathrm{PM}^{4}$ para Artibeus, como discutido anteriormente. Todos os quatro critérios de reconhecimento de um atavismo (HALL 1984) enquadram-se no caso deste pré-molar. Nenhuma espécie de Artibeus possui os $\mathrm{PM}^{2}$ e, tomando-se uma filogenia que inclua gêneros mais próximos a Artibeus como Chiroderma Peters, 1860 e Uroderma Peters, 1866 (Bussche et al. 1998), ou ainda filogenias que incluam todos os gêneros da família Phyllostomidae (WETTERER et al. 2000), verifica-se que esse dente não ocorre em nenhum gênero. Como exceção, no gênero Anoura Gray, 1838 ocorre o $\mathrm{PM}^{2}$, o que é considerado uma autapomorfia (WETTERER et al. 2000), sem nenhuma relação com Artibeus. Isto leva a crer que esse atavismo remonta a um período muito mais antigo, anterior à diversificação da família Phyllostomidae. Em algumas famílias recentes, como Hipposideridae e Rhinolophidae, e em alguns gêneros como Miniopterus Bonaparte, 1837, Myotis
Kaup, 1829 (Vespertilionidae), Natalus Gray, 1838 (Natalidae) e Thyroptera Spix, 1823 (Thyropteridae) ocorre o $\mathrm{PM}^{2}$ reduzido, assim como em alguns grupos fósseis de quirópteros (comunicação pessoal Bernard Sigé).

\section{Agênese do terceiro molar inferior}

Casos de agênese dentária são amplamente relatados na literatura mastozoológica para as mais diversas ordens (Hoff \& Hoff 1996, Vila et al. 1993, Suchentrunk et al. 1992). Estas se caracterizam pela ausência completa dos dentes, sem nenhum resquício dos alvéolos, diferentemente das perdas patológicas (normalmente de origem traumática), onde pequenos traços dos alvéolos em processo de fechamento podem ainda ser encontrados (Drehmer \& Ferigolo 1996). Na figura 6 pode-se observar a mandíbula do exemplar A. lituratus DZMAM 100, onde o terceiro molar do lado direito foi perdido e o alvéolo está aberto.

O crescimento e a morfogênese dentária em mamíferos têm sido relacionados à expressão de certos genes como o "sonic hedgehog" (DAssule et al. 2000), conseqüentemente é possível que as agêneses dentárias tenham relação direta com os mecanismos de regulação e expressão desses genes.

De um total de 13 trabalhos descrevendo a fórmula dentária de $A$. lituratus, em quatro deles há relatos de agênese dos terceiros molares inferiores, sempre em baixas freqüências (D.R. Patten 1971, dados não publicados, Davis 1984, RamírezPulido \& Müdespacher 1987, presente trabalho). Assim como há casos de agênese deste dente para A. fraterculus (D.R. Patten 1971, dados não publicados), A. jamaicensis (DAvis 1970, JONES Jr. 1978, Taboada 1979) e A. hirsutus (ANDERson in Webster \& Jones Jr. 1983) (Tab. I). Porém, os dados relativos à ausência do terceiro molar inferior devem estar subestimados na literatura, já que muitos autores referem-se somente aos molares superiores, que são considerados mais informativos em termos sistemáticos.

\section{Variação numérica dos terceiros molares em Artibeus (Artibeus)}

A questão da variação na fórmula dentária quanto aos terceiros molares superiores e inferiores no gênero Artibeus (subgênero Artibeus) merece uma análise mais detalhada do ponto de vista evolutivo e filogenético. Como já foi citada, esta questão sempre foi abordada do ponto de vista sistemático, sem consenso sobre a utilidade ou não deste caráter. Para a análise foi realizada uma compilação de todas as informações disponíveis na literatura sobre o assunto (Tab. I).

Das nove espécies do subgênero Artibeus, apenas para $A$. amplus não foram encontradas informações sobre variação de fórmula dentária. Artibeus amplus foi descrito recentemente (HANDLEy 1987) e tem distribuição geográfica restrita ao norte da América do Sul, incluindo Colômbia, Venezuela, Guiana e Suriname (Lim et al. 2003). Essa é a única espécie de Artibeus (Artibeus) com três molares superiores e inferiores fixos (MARQUES-AgUiAR 1994), porém nenhum trabalho foi realizado abordando a questão da fórmula dentária e não está descartada a hipótese de que possam ocorrer variações no número de molares desta espécie também. 
As informações obtidas na literatura (Tab. I) revelam que as variações quanto à presença do terceiro molar superior podem ser enquadradas em dois diferentes tipos: 1) variação geográfica, ou seja, uma mesma espécie possui diferenças neste caráter em populações que estão separadas geograficamente; 2) variação intrapopulacional, que são as variações neste caráter entre indivíduos de uma mesma população. Um exemplo ilustrativo de variação geográfica ocorre em A. jamaicensis, cujos indivíduos das populações do leste dos Andes possuem três molares superiores e os do oeste dos Andes e América Central possuem apenas dois (HANDley 1987). Para o Brasil, há um caso descrito de variação geográfica em $A$. obscurus, cuja população do leste do país, de São Paulo até a Paraíba, possuí dois molares superiores e, a do oeste da Amazônia, possuí três (TADDEI et al. 1998) (Tab. I). A variação intrapopulacional engloba todos os demais casos de variação de ocorrência de terceiros molares superiores, inclusive os casos de atavismo descritos para $A$. lituratus e A. fimbriatus. Como pode ser observado na tabela I, este tipo de variação é bastante comum e há diferenças nas freqüências de presença ou ausência dos terceiros molares superiores em diferentes espécies e em diferentes populações de uma mesma espécie.

Quanto ao terceiro molar inferior, há vários casos de agênese descritos na literatura, porém não há indícios de que ocorram variações geográficas. A agênese ocorre sempre em baixa freqüência e já foi registrada para diferentes espécies em diferentes locais (Tab. I). DAvis (1984) afirma que em A. lituratus no México e na América Central o terceiro molar inferior não ocorre em um ou ambos os lados da mandíbula em cerca de $2 \%$ da população. Este é o único trabalho que cita a freqüência de ocorrência de agênese em uma população (Tab. I). Porém, a maior freqüência de ocorrência de agênese do terceiro molar inferior ocorre na população de A. jamaicensis da Ilha de Cuba, onde cerca de 7\% (10/146) dos indivíduos não possuem estes dentes (TABOADA 1979). Provavelmente, esta alta incidência da agênese está relacionada ao fato desta população estar restrita a uma ilha de pequenas proporções.

As espécies com menor variação quanto aos terceiros molares são $A$. fimbriatus, $A$. fraterculus (apenas uma citação) e A. lituratus (Tab. I). Em A. fimbriatus, há apenas um caso de $\mathrm{M}^{3}$ extranumerário (Sérgio Althoff com. pes.), em A. fraterculus um caso de agênese do $M_{3}$ (D.R. Patten 1971, dados não publicados) e em $A$. lituratus seis casos de agênese do $\mathrm{M}_{3}$ e sete de $\mathrm{M}^{3}$ extranumerários. Por outro lado, para $A$. hirsutus, $A$. inopinatus (com apenas um trabalho cada um se referindo a este assunto), A. jamaicensis, A. obscurus e A. planirostris há dados de literatura que comprovam a ampla variação deste caráter (Tab. I).

A ampla variação quanto à presença do terceiro molar superior e inferior em Artibeus (Artibeus) é um polimorfismo, ou seja, a existência de variação fenotípica dentro de uma população, seja ela ou não de base genética (Futuyma 2002). No caso de Artibeus, esta variação fenotípica populacional também se estende a nível geográfico. A possibilidade de esta vari- ação ser devido a um polimorfismo já tinha sido sugerida por PAtterson et al. (1992: 198), quando comenta das dificuldades na identificação e delimitação de espécies de Artibeus.

Analisando-se a questão dos terceiros molares, tanto superiores quanto inferiores no gênero Artibeus, constata-se vários indícios que revelam uma tendência à perda destas estruturas. O terceiro molar superior, e em menor grau também o inferior, é sempre um dente muito reduzido e simplificado, sem maior participação na função mastigatória, ou seja, sua presença ou ausência não tem nenhum significado funcional/ adaptativo. Essa morfologia corrobora uma tendência evolutiva amplamente conhecida para a dentição dos mamíferos em geral, como atesta Wolsan (1984: 129), "the progressive simplification in shape and simultaneous reduction in size of a tooth have clearly preceded its loss". Os terceiros molares no subgênero Artibeus também podem ser considerados estruturas vestigiais. Segundo HaLl (2003), vestígios "são remanescentes evolutivos de uma característica ancestral... caracterizados como não funcionais e... presumivelmente não adaptativos, sempre em adultos".

Outro indício de que o processo evolutivo de perda dos terceiros molares esteja ocorrendo é o fato de que em algumas espécies do subgênero Artibeus, como A. lituratus, A. fimbriatus e $A$. fraterculus, estes dentes já não ocorrem, a não ser em raros casos de atavismo, como o relatado aqui para A. lituratus. Além disso, em praticamente todas as demais espécies do subgênero a presença do terceiro molar superior não é uma característica fixa. Já nas espécies do subgênero Dermanura, os terceiros molares superiores e inferiores não ocorrem na maioria das espécies (MuÑoz 1995), e em Enchistenes e Koopmania, estes dentes são reduzidos em relação aos segundos molares superiores e inferiores (Marques-Aguiar 1994). Ou seja, em todo os membros do gênero Artibeus existe uma clara tendência à redução/ perda dos terceiros molares.

Considerando-se a ampla variação observada nos terceiros molares de espécies de Artibeus (subgênero Artibeus), tanto em nível populacional quanto geográfico, não é recomendável sua utilização como um caráter sistemático para a separação de espécies. A utilização isolada deste caráter pode levar a identificações errôneas. Independente de sua utilidade na sistemática, os padrões de variação dos terceiros molares em Artibeus constituem um interessante objeto de estudo de processos evolutivos, sendo que amostras maiores, advindas de outras regiões, poderão fornecer uma base mais ampla para futuras comparações e análises.

\section{AGRADECIMENTOS}

Agradecemos a Márcio Borges Martins, curador da coleção de mamíferos do Museu Ciências Naturais da FZB-RS, e a Hamilton Zanardi Grillo, curador da coleção de mamíferos do Museu de Ciências Naturais da UNIVATES, que nos permitiram examinar exemplares depositados nas coleções científicas 
de suas instituições. A Cristiano Schwertner pelo auxílio na confecção das fotos dos crânios e ao Laboratório de Entomologia Sistemática, Departamento de Zoologia, UFRGS pelo empréstimo do equipamento fotográfico e óptico. Ao Dr. Bernard Sigé que discutiu questões evolutivas e nos forneceu dados sobre variação morfológica. À Dra. Marta E. Fabián, à Dra. Susi Missel Pacheco e à Msc. Rosane Vera Marques pela leitura e sugestões ao manuscrito. A autora agradece ao CNPq pela concessão da Bolsa Recém-Doutor (Processo 302480/021), que possibilitou a execução deste trabalho.

\section{REFERÊNCIAS BIBLIOGRÁFICAS}

Anderson, S.; K.F. Koopman \& G.K. Creighton. 1982. Bats of Bolivia: an annotated checklist. American Museum Novitates, New York, 2750: 1-24.

Astúa de Moraes, D.; B. Lemos \& R. Cerqueira. 2001. Supernumerary molars in neotropical opossums (Didelphimorphia, Didelphidae). Zeitschrift fur Saugetierkunde, Berlin, 66: 193-203.

Barquez, R.M.; M.A. Mares \& J.K. Braun. 1999. The bats of Argentina. Special Publications Museum of Texas Tech University, Lubbock, 42: 1-275.

BejDER, L. \& B.K. HALL. 2002. Limbs in whales and limblessness in other vertebrates: mechanisms of evolutionary and developmental transformation and loss. Evolution and Development, Malden, 4 (6): 445-458.

Bussche, R.A. van den; J.L. Hudgeons \& R.J. Baker. 1998. Phylogenetic accuracy, stability, and congruence: relationships within and among the New World bat genera Artibeus, Dermanura and Koopmania, p. 59-71. In: T.H. Kunz \& P.A. RACEY (Eds). Bat biology and conservation. Washington, Smithsonian Institution Press, 365p.

Dassule, H.R.; P. Lewis; M. Bei; R. MaAs \& A.P. Mcmahon. 2000. Sonic hedgehog regulates growth and morphogenesis of the tooth. Development, Cambridge, 127: 4775-4785.

DAvis, W.B. 1970. The large fruit bats (genus Artibeus) of Middle America, with a review of the Artibeus jamaicensis complex. Journal of Mammalogy, Lawrence, 51 (1): 105-122.

- 1984. Review of the large fruit-eating bats of the Artibeus "lituratus" complex (Chiroptera: Phyllostomidae) in Middle America. Occasional Papers the Museum Texas Tech University, Lubbock, 93: 1-16.

Dias, D.; A.L. Peracchi \& S.S.P. Da Silva. 2002. Quirópteros do Parque Estadual da Pedra Branca, Rio de Janeiro, Brasil (Mammalia, Chiroptera). Revista Brasileira de Zoologia, Curitiba, 19 (Supl. 2): 113-140.

Drehmer, C.J. \& J. Ferigolo. 1996. Anomalias e patologias dentárias em Arctocephalus G. Saint-Hilaire \& Cuvier (Pinnipedia, Otariidae) da costa do Rio Grande do Sul, Brasil. Revista Brasileira de Zoologia, Curitiba, 13 (4): 857 865.

Eisenberg, J.F. \& K.H. Redford. 1999. Mammals of the Neotropics. The Central Neotropics: Ecuador, Peru, Bolivia,
Brazil. Chicago, The University of Chicago Press, vol. 3, 609p.

Futuyma, D.J. 2002. Evolutionary Biology. Sunderland, Sinauer Associates, $3^{\text {th }}$ ed., 763p.

Gardner, A.L. 1977. Feeding habits, p. 293-350. In: R.J. BAKER; J. Knox Jones Jr. \& D.C. Carter (Eds). Biology of bats of the New World Family Phyllostomidae. Part II. Lubbock, Special Publications the Museum Texas Tech University, $364 p$.

Gould, S.J. 1982. A galinha e seus dentes. Rio de Janeiro, Paz e Terra, 404p.

HaLl, B.K. 1984. Developmental mechanisms underlying the formation of atavisms. Biological Reviews of the Cambridge Philosophical Society, Cambridge, 59: 89-124.

. 2003. Descent with modification: the unity underlying homology and homoplasy as seen through an analysis of development and evolution. Biological Reviews of the Cambridge Philosophical Society, Cambridge, 78: 409-433.

HandLeY JR., C.O. 1987. New species of mammals from northern south America: fruit-eating bats, genus Artibeus Leach. In: B.D. Patterson \& R.M. Timm (Eds). Studies in Neotropical Mammalogy: Essays in Honor Philip Hershkovitz. Fieldiana Zoology, n.s., Chicago, 1382 (39): 163-172.

. 1989. The Artibeus of Gray 1838, p. 443-468. In: K.H. Redford \& J.F. Eisenberg (Eds). Advances in Neotropical Mammalogy. Gainesville, The Sandhill Crane Press, 614p. Hoff, G.L. \& D.M. Hoff. 1996. Dental anomalies in mammals, p. 100-108. In: A. Fairbrother; L.N. Locke \& G.L. Hoff (Eds). Noninfectious diseases of wildlife. Des Moines, Iowa State University Press, XIII+219p.

Jones Jr., J.K. 1978. A new bat of the genus Artibeus from the Lesser Antillean Island of St. Vincent. Occasional Papers the Museum Texas Tech University, Lubbock, 51: 1-6.

Koepcke, J. \& R. KRAfT. 1984. Cranial and external characters of the large fruit bats of the genus Artibeus from Amazonian Peru. Spixiana, München, 7 (1): 75-84.

Koopman, K.F. 1993. Order Chiroptera, p. 137-241. In: D.E. WILSON \& D.M. REEDER (Eds.). Mammal species of the world: a taxonomic and geographic reference. Washington, D.C., Smithsonian Institution Press, $2^{\text {nd }}$ ed., XVIII+1206p.

Lim, B.K.; H.H. Genoways \& M.D. Engstrom. 2003. Results of the Alcoa Foundation-Suriname Expeditions. XII. First record of the giant fruit-eating bat, A. amplus, (Mammalia: Chiroptera) from Suriname with a review of the species. Annals of the Carnegie Museum, Pittsburgh, 72 (2): 99-107.

Marques-Aguiar, S.A. 1994. A systematic review of the large species of Artibeus Leach, 1821 (Mammalia: Chiroptera), with some phylogenetic inferences. Boletim do Museu Paraense Emílio Goeldi, Série Zoologia, Belém, 10 (1): 3-83.

Menu, H.; S. Hand \& B. Sigé. 2002. Oldest Australian vespertilionid (Microchiroptera) from the early Miocene of Riversleigh, Queensland. Alcheringa, Sydney, 26: 319-331.

Myers, P. \& R.M. Wetzel. 1979. New records of mammals from

Revista Brasileira de Zoologia 21 (3): 639-648, setembro 2004 
Paraguay. Journal of Mammalogy, Lawrence, 60 (3): 638641.

MuÑoz, J. 1995. Clave de murciélagos vivientes em Colombia. Medellín, Editorial Universidad de Antioquia, 132p.

OwEn, R.D. 1987. Phylogenetic analyses of the bat subfamily Stenodermatinae (Mammalia: Chiroptera). Special Publications the Museum Texas Tech University, Lubbock, 26: 1-65.

. 1991. The systematic status of Dermanura concolor

(Peters, 1865) (Chiroptera: Phyllostomidae), with description of a new genus. Bulletin American Museum of Natural History, New York, 206: 18-25.

Patterson, B.D.; V. Pacheco \& M.V. Ashley. 1992. On the origins of the western slope region of endemism: systematics of fig-eating bats, genus Artibeus. Memorias del Museo de Historia Natural, U.N.M.S.M., Lima, 21: 189-205.

Paula Couto, C. 1979. Tratado de Paleomastozoologia. Academia Brasileira de Ciências, Rio de Janeiro, 590p.

Ramírez-Pulido, J. \& C. Müdespacher. 1987. Formulas dentarias anormales en algunos murcielagos mexicanos. Acta Zoologica Mexicana, Cidade do México, 23: 1-54.

RuI, A.M. \& M.E. FabIÁn. 1997. Quirópteros de la familia Phyllostomidae (Mammalia, Chiroptera) em selvas del estado de Rio Grande do Sul, Brasil. Chiroptera Neotropical, Brasília, 3 (2): 75-77.

Sigé, B.; J. Habersetzer \& G. Storch. 1998. The deciduous dentition and dental replacement in the Eocene bat Palaeochiropteryx tupaiodon from Messel: the primitive condition and beginning of specialization of milk teeth among Chiroptera. Lethaia, Oslo, 31: 349-358.

Simmons, N.B. \& R.S. Voss. 1998. The mammals of Paracou, French Guiana: a neotropical lowland rainforest fauna. Part 1. Bats. Bulletin of the American Museum of Natural History, New York, 237: 1-219.

STiassny, M.L.J. 1992. Atavisms, phylogenetic character reversals, and the origin of evolutionary novelties. Netherlands Journal of Zoology, Leidn, 42: 260-276.

. 2002. Atavisms, p. 10-14. In: B.K. Hall \& W.M. OlsEN (Eds). Key Concepts and Approaches in Evolutionary

Recebido em 05.I.2004; aceito em 30.VII.2004.
Developmental Biology. Cambridge, Harvard University Press, 476p.

Suchentrunk, F.; J. Markowski; T. Janiszewski \& G.B. Hartl. 1992. Dental and cranial anomalies in Austrian and Polish brown hare Lepus europaeus populations. Acta Theriologica, Warszawa, 37 (3): 241-257.

TABOADA, G.S. 1979. Los murciélagos de Cuba. Cuba, Editorial Academia, Editora de la Academia de Ciencias de Cuba, 423p.

TAddei, V.A. 1979. Phyllostomidae (Chiroptera) do norte-ocidental do estado de São Paulo. III- Stenodermatinae. Ciência e Cultura, São Paulo, 31 (8): 900-914.

Taddei, V.A.; C.A. Nobile \& E. Morielle-Versute. 1998. Distribuição geográfica e análise morfométrica comparativa em Artibeus obscurus (Schinz, 1821) e Artibeus fimbriatus Gray, 1838 (Mammalia, Chiroptera, Phyllostomidae). Ensaios e Ciência, Campo Grande, 2 (2): 71-127.

Vilà, C.; V. Urios \& J. Castroviejo. 1993. Tooth losses and anomalies in the wolf (Canis lupus). Canadian Journal of Zoology, Ottawa, 71: 968-971.

Webster, W.D. \& J.K. Jones JR. 1980. Noteworthy records of bats from Bolivia. Occasional Papers the Museum Texas Tech University, Lubbock, 68: 1-6.

-1983. Artibeus hirsutus and Artibeus inopinatus. Mammalian Species, New York, 199: 1-3.

WeDERLIN, L. 1987. Supernumerary teeth in Lynx lynx and the irreversibility of evolution. Journal of Zoology, London, 211: 259-266.

Wetterer, A.L.; M.V. Rockman \& N.B. Simmons. 2000. Phylogeny of phyllostomid bats (Mammalia: Chiroptera): data from diverse morphological systems, sex chromosomes and restriction sites. Bulletin of the American Museum of Natural History, New York, 248: 1-200.

Wolsan, M. 1984. The origin of extra teeth in mammals. Acta Theriologica, Warszawa, 29 (10): 128-133.

ZIMA, J. 1988. Incidence of dental anomalies in Capreolus capreolus from Czechoslovakia. Folia Zoologica, Prague, 37 (2): 129-144. 